\title{
Blind Block Synchronization Algorithms in Cyclic Prefix Systems
}

\author{
Borching $\mathrm{Su}$ \\ Department of Electrical Engineering \\ California Institute of Technology \\ Pasadena, California 91125 \\ Email: borching@systems.caltech.edu
}

\author{
P. P. Vaidyanathan \\ Department of Electrical Engineering \\ California Institute of Technology \\ Pasadena, California 91125 \\ Email: ppvnath@systems.caltech.edu
}

\begin{abstract}
In orthogonal frequency division multiplexing (OFDM) systems, symbol synchronization is a critical step for successful data transmission. While this task is done in most current systems by using training symbols, a few studies have been dedicated to solving the problem blindly, that is, where training symbols are not available. Blind symbol synchronization problem is especially important in many blind channel estimation algorithms in the literature which assume that OFDM symbol synchronization is perfect. In this paper, a broader version of the blind symbol synchronization problem is studied, namely, blind block synchronization in cyclic-prefix (CP) systems. The proposed algorithm for this broader problem covers the blind symbol synchronization problem in OFDM systems. Unlike previously reported algorithms which are based on obtaining sufficient statistics of received samples, the proposed algorithm is capable of identifying the correct block boundaries using much less received data in absence of noise. Simulation results of the proposed algorithm not only verify the declared property but also demonstrate improvement in accuracy of symbol synchronization over previously reported algorithms in presence of noise. ${ }^{1}$
\end{abstract}

\section{INTRODUCTION}

Blind channel identification in cyclic prefix systems has been studied extensively in the literature [1], [4]. Besides a constant bandwidth overhead introduced in each block, a blind channel estimation method usually requires very little extra bandwidth to perform channel estimation. Most existing blind estimation methods assume the boundaries of blocks of the received stream are perfectly known to the receiver. In practical applications, however, this assumption is usually not true since no extra known samples are transmitted. In this paper we study the problem of blind recovery of block boundaries for the received signal.

A number of blind block synchronization algorithms in cyclic prefix-based orthogonal frequency division multiplexing (OFDM) have been developed in the past [2], [5]-[8]. These methods, however, require a large number of received data for successful block synchronization. Our proposed algorithm possesses two advantages over previously reported methods: 1) In absence of noise, the proposed algorithm guarantees correct recovery of block boundaries using only three received blocks whereas all previously reported algorithms do not work properly when the number of received blocks is smaller than the block size. This makes the proposed algorithm much more promising for fast-varying channels. 2) When the noise is present, simulation results as reported in Section IV show that with the same amount of received data, the proposed algorithm has an obvious improvement in blind block synchronization error rate performance over the algorithm proposed in [2].

The rest of the paper will be organized as follows. In Section II the problems of interest, namely the blind block

${ }^{1}$ Work supported in parts by the NSF grant CCF-0428326 and the Moore Fellowship of the California Institute of Technology.

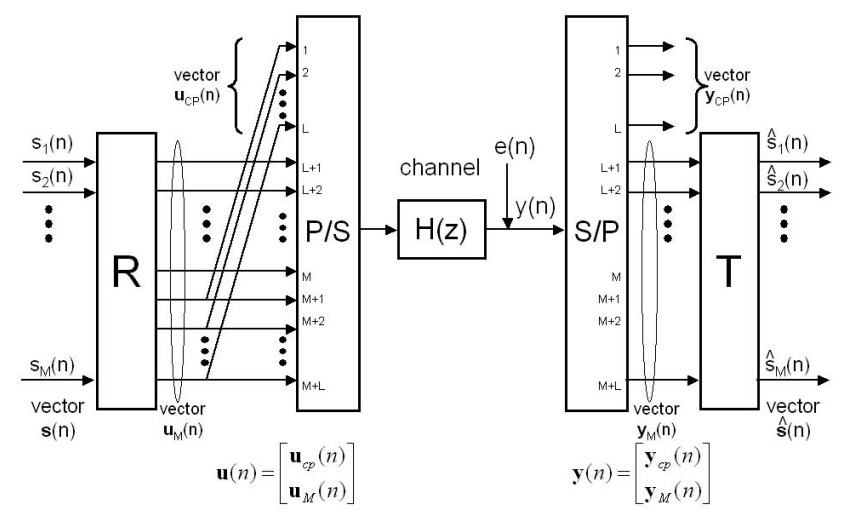

Fig. 1. A typical cyclic prefix system.

synchronization problems in cyclic prefix systems, will be formulated. In Section III, the proposed blind block synchronization algorithm will be presented. In Section IV, simulation results are provided to evaluate the system performance of the proposed algorithm and to compare it with that of a previously reported algorithm. Finally, the conclusions are made in Section V.

\section{A. Notations}

Boldfaced lower case letters represent column vectors. Boldfaced upper case letters and calligraphic upper case letters are reserved for matrices. Superscripts ${ }^{*},{ }^{T}$, and ${ }^{\dagger}$ as in $a^{*}$, $\mathbf{A}^{T}$, and $\mathbf{A}^{\dagger}$ denote the conjugate, transpose, and transposeconjugate operations, respectively. All the vectors and matrices in this paper are complex-valued. $\mathbf{I}_{M}$ is the $M \times M$ identity matrix, and $\mathbf{0}_{m \times n}$ is the $m \times n$ zero matrix.

Due to special properties of cyclic prefixes, we will use the following notation extensively in this paper. Suppose $\mathbf{y}$ is an $m \times 1$ column vector $\mathbf{y}=\left[\begin{array}{llll}y_{1} & y_{2} & \cdots & y_{m}\end{array}\right]^{T}$. Then the notation $[\mathbf{y}]_{b}^{a}$ denotes the $(b-a+1) \times 1$ vector

$$
[\mathbf{y}]_{b}^{a}=\left[\begin{array}{llll}
y_{a} & y_{a+1} & \cdots & y_{b}
\end{array}\right]^{T}
$$

if $1 \leq a \leq b \leq m$. An extension of this definition to any arbitrary pair of integers $a$ and $b$ satisfying $a \leq b$ is made by defining $y_{k}$ as $\left.y_{(k-1} \bmod m\right)+1$ for any $k>$ $m$ or $k<1$. For example, if $\mathbf{y}=\left[\begin{array}{lll}y_{1} & y_{2} & y_{3}\end{array}\right]^{T}$, $a=-1$, and $b=7$, then $[\mathbf{y}]_{b}^{a}$ denotes the vector $\left[\begin{array}{lllllllll}y_{2} & y_{3} & y_{1} & y_{2} & y_{3} & y_{1} & y_{2} & y_{3} & y_{1}\end{array}\right]^{T}$.

\section{Problem Formulation}

\section{A. Cyclic Prefix System Overview}

Consider the communication system using cyclic prefix $(\mathrm{CP})$ depicted in Fig. 1. The source symbols $s_{1}(n), s_{2}(n), \ldots, s_{M}(n)$ 
may come from $M$ different users or from a serial-to-parallel operation on data of a single user. For convenience we consider the blocked version $\mathbf{s}(n)$ as indicated. The vector $\mathbf{s}(n)$ is precoded by an $M \times M$ constant matrix $\mathbf{R}$ and results in precoded data $\mathbf{u}_{M}(n)$. In particular, for OFDM or multi-carrier (MC) systems, $\mathbf{R}$ is the normalized IDFT matrix; for singlecarrier cyclic prefix (SC-CP) systems, $\mathbf{R}$ is chosen as $\mathbf{I}_{M}$. A cyclic prefix of length $L$, taken from the last $L$ elements of $\mathbf{u}_{M}(n)$, is defined as $\mathbf{u}_{c p}(n)=\left[\begin{array}{ll}\mathbf{0}_{L \times(M-L)} & \mathbf{I}_{L}\end{array}\right] \mathbf{u}_{M}(n)$. We assume $L+1<M$. The cyclic prefix is appended to $\mathbf{u}_{M}(n)$, forming a vector

$$
\mathbf{u}(n)=\left[\begin{array}{ll}
\mathbf{u}_{c p}(n)^{T} & \mathbf{u}_{M}(n)^{T}
\end{array}\right]^{T}=\left[\mathbf{u}_{M}(n)\right]_{M}^{-L+1}
$$

whose length is $P=M+L$. The vector $\mathbf{u}(n)$, after parallelto-serial conversion, is sent over the channel $H(z)$. We assume $H(z)$ is an FIR channel with a maximum order $L$, i.e.,

$$
H(z)=\sum_{k=0}^{L_{0}} h_{k} z^{-k}
$$

where $L_{0} \leq L$. Define $\mathbf{h}$ as the $(L+1)$-vector $\left[\begin{array}{llll}h_{0} & h_{1} & \cdots & h_{L}\end{array}\right]^{T}$ where the values of $h_{L_{0}+1}, \ldots, h_{L}$ are set to zeros if $L>L_{0}$. The signal at the channel output is corrupted by an additive white Gaussion noise $e(n)$. The received samples $y(n)$ are blocked into $P \times 1$ vectors $\mathbf{y}(n)$.

For a moment assume perfect block synchronization between the transmitter and receiver. Let

$$
\mathbf{y}(n)=\left[\begin{array}{llll}
y(n P) & y(n P+1) & \cdots & y(n P+P-1)
\end{array}\right]^{T}
$$

and denote $\mathbf{y}_{c p}(n)$ as the first $L$ entries and $\mathbf{y}_{M}(n)$ as the last $M$ entries of $\mathbf{y}(n)$ so that $\mathbf{y}(n)=\left[\begin{array}{ll}\mathbf{y}_{c p}(n)^{T} & \mathbf{y}_{M}(n)^{T}\end{array}\right]^{T}$. Also let $\mathbf{e}(n)=\left[\begin{array}{ll}\mathbf{e}_{c p}(n)^{T} & \mathbf{e}_{M}(n)^{T}\end{array}\right]^{T}$ denote the blocked version of the noise $e(n)$. It can be shown that

$$
\mathbf{y}_{M}(n)=\mathbf{H}_{c i r} \mathbf{u}_{M}(n)+\mathbf{e}_{M}(n)
$$

where $\mathbf{H}_{c i r}$ is an $M \times M$ circulant matrix [9] whose first column is $\left[\begin{array}{llllll}h_{0} & \cdots & h_{L} & 0 & \cdots & 0\end{array}\right]^{T}$. The $L \times 1$ vector $\mathbf{y}_{c p}(n)$ contains inter-block interference (IBI) and can be expressed as

$$
\mathbf{y}_{c p}(n)=\mathbf{H}_{l} \mathbf{u}_{c p}(n)+\mathbf{H}_{u} \mathbf{u}_{c p}(n-1)+\mathbf{e}_{c p}(n)
$$

where

$$
\mathbf{H}_{l} \triangleq\left[\begin{array}{ccc}
h_{0} & & \mathbf{0} \\
\vdots & \ddots & \\
h_{L-1} & \cdots & h_{0}
\end{array}\right] \text { and } \mathbf{H}_{u} \triangleq\left[\begin{array}{ccc}
h_{L} & \cdots & h_{1} \\
& \ddots & \vdots \\
\mathbf{0} & & h_{L}
\end{array}\right]
$$

are $L \times L$ matrices.

\section{B. Problem Statement}

The assumption of block synchronization between the transmitter and receiver, however, is usually not true in practice. Suppose the blocking is performed with a timing mismatch $d \in[-P / 2, P / 2)$. Then the samples collected in the $n$th block will be

$$
\begin{aligned}
\mathbf{y}^{(d)}(n) & =\left[\begin{array}{llll}
y(n P+d) & y(n P+d+1) & \cdots & y(n P+d+P-1)
\end{array}\right. \\
& \left.=\left[\begin{array}{lll}
{\left[\begin{array}{l}
\mathbf{y}_{c p}^{(d)}(n)
\end{array}\right]^{T}} & {\left[\mathbf{y}_{M}^{(d)}(n)\right.}
\end{array}\right]^{T}\right]^{T}
\end{aligned}
$$

The problem of block synchronization can be described as follows. Given the received sample stream $y(n)$, with a possible unknown timing offset with respect to the transmitter, how do we determine the optimal $d \in[-P / 2, P / 2)$ that represents the starting index of a received block? The block synchronization problem is a broader version of the "timing synchronization" problem or the "symbol synchronization" problem in OFDM systems, in which the precoder $\mathbf{R}$ of the $\mathrm{CP}$ system is chosen as the IDFT matrix. Without loss of generality and for convenience of the presentation, we assume the "correct answer" is always $d=0$. Furthermore, when the effective channel order $L_{0}$ is strictly smaller than the cyclic prefix length $L$, we observe that $d=-L+L_{0},-L+$ $L_{0}+1, \ldots, 0$ can all be considered "correct answers" since the equivalent channel vector is

$$
\mathbf{h}^{(d)}=\left[\begin{array}{lllll}
\mathbf{0}_{1 \times(-d)} & h_{0} & \cdots & h_{L_{0}} & \mathbf{0}_{1 \times\left(L-L_{0}+d\right)}
\end{array}\right]^{T}
$$

and the first $M$ elements of $\mathbf{y}^{(d)}(n)$ are free from inter-block interference.

\section{Proposed Method}

The proposed approach to the blind block synchronization problem is derived from an existing blind channel estimation algorithm proposed in [1]. We first consider the situation where the noise is absent. Define a "composite block" whose elements are chosen from two consecutive received blocks:

$$
\overline{\mathbf{y}}(n)=\left[\begin{array}{lll}
\mathbf{y}_{M}(n-1) & \mathbf{y}_{c p}(n) & \mathbf{y}_{M}(n)
\end{array}\right]^{T} .
$$

It is readily verified that $\overline{\mathbf{y}}(n)=\tilde{\mathbf{H}} \tilde{\mathbf{u}}(n)$ where

$$
\tilde{\mathbf{H}}=\left[\begin{array}{c|c|c|c}
\multicolumn{2}{|c|}{\mathbf{H}_{c i r}} & \multicolumn{2}{|c}{\mathbf{0}_{M \times M}} \\
\hline\left[\mathbf{0}_{L \times(M-L)}\right. & \left.\mathbf{H}_{u}\right] & \mathbf{0}_{L \times(M-L)} & \left.\mathbf{H}_{l}\right] \\
\hline \mathbf{0}_{M \times M} & \multicolumn{2}{|c|}{\mathbf{H}_{c i r}} \\
\hline
\end{array}\right]
$$

and $\tilde{\mathbf{u}}(n)=\left[\begin{array}{cc}\mathbf{u}_{M}(n-1)^{T} & \mathbf{u}_{M}(n)^{T}\end{array}\right]^{T}$. Note that here $\tilde{\mathbf{H}}$ has a size of $(2 M+L) \times 2 M$. This means each composite block, $\overline{\mathbf{y}}(n)$, of size $2 M+L$, is a linear combination of $2 M$ columns of $\tilde{\mathbf{H}}$, and is always confined in a $2 M$-dimension subspace. This special property, however, is no longer true when the block synchronization is not correct. This observation constitutes the basic idea of the proposed method for blind block synchronization.

Furthermore, each received composite block $\overline{\mathbf{y}}(n)$ can be reformulated into a $Q$-column matrix $\mathbf{Y}_{Q}(n)$ as defined below. Here, $Q$ can be chosen as any positive integer and is called the repetition index [1].

$$
\mathbf{Y}_{Q}(n)=\left[\begin{array}{llll}
\overline{\mathbf{y}}_{0, Q-1}(n) & \overline{\mathbf{y}}_{1, Q-2}(n) & \cdots & \overline{\mathbf{y}}_{Q-1,0}(n)
\end{array}\right]
$$
where each column is a $(2 M+L+Q-1)$-vector defined as $\left.\overline{\mathbf{y}}_{k l}(n)=\left[\begin{array}{lll}{\left[\mathbf{y}_{M}(n-1)\right.} & ]_{M}^{-k+1}\end{array}\right]^{T} \quad \mathbf{y}_{c p}(n)^{T} \quad\left[\begin{array}{ll}\mathbf{y}_{M}(n) & ]_{M+l}^{1}\end{array}\right]^{T}\right]^{T}$. When block synchronization between the transmitter and the receiver is perfect, it can be shown that

$$
\mathbf{Y}_{Q}(n)=\overline{\mathbf{H}}_{Q} \mathbf{U}_{Q}(n)
$$

where $\overline{\mathbf{H}}_{Q}$ and $\mathbf{U}_{Q}(n)$ are defined as follows.

$$
{ }_{T}^{T} \overline{\mathbf{H}}_{Q}=\left[\begin{array}{ccc}
\mathbf{H}_{c i r} & \mathbf{0}_{M \times(M+Q-1)} \\
\mathbf{0}_{(L+Q-1) \times(M-L)} & \mathcal{H}_{L+Q-1} & \mathbf{0}_{(L+Q-1) \times(M-L)} \\
\mathbf{0}_{M \times(M+Q-1)} & & \mathbf{H}_{c i r 2}
\end{array}\right]
$$

where $\mathbf{H}_{c i r 2}$ is obtained by moving the first $L$ rows of $\mathbf{H}_{\text {cir }}$ to the bottom and $\mathcal{H}_{L+Q-1}$ is a $(L+Q-1) \times(2 L+Q-1)$ Toeplitz matrix whose first row is $\left[\begin{array}{lllllll}h_{L} & \cdots & h_{0} & 0 & \cdots & 0\end{array}\right]$ and whose first column is $\left[\begin{array}{llll}h_{L} & 0 & \cdots & 0\end{array}\right]^{T}$.

$$
\mathbf{U}_{Q}(n)=\left[\begin{array}{llll}
\overline{\mathbf{u}}_{0, Q-1}(n) & \overline{\mathbf{u}}_{1, Q-2}(n) & \cdots & \overline{\mathbf{u}}_{Q-1,0}(n)
\end{array}\right]
$$

where 


$$
\overline{\mathbf{u}}_{k l}(n)=\left[\begin{array}{ll}
\left.\mathbf{u}_{M}(n-1)\right]_{M}^{-k+1^{T}} & {\left[\mathbf{u}_{M}(n)\right]_{M+l-L}^{-L+1}{ }^{T}}
\end{array}\right]^{T} .
$$

Note that $\overline{\mathbf{H}}_{Q}$ is a tall matrix with size $(2 M+Q+L-$ $1) \times(2 M+Q-1)$. So each column of $\mathbf{Y}_{Q}(n)$ is confined in a $(2 M+Q-1)$-dimension subspace. Now, suppose $J$ consecutive received blocks $\mathbf{y}(n), n=0,1, \ldots, J-1$, are available. Consider the $(2 M+L+Q-1) \times(J-1) Q$ matrix

$$
\mathbf{Y}_{J, Q}=\left[\begin{array}{llll}
\mathbf{Y}_{Q}(1) & \mathbf{Y}_{Q}(2) & \cdots & \mathbf{Y}_{Q}(J-1)
\end{array}\right] .
$$

It is readily verified that

$$
\mathbf{Y}_{J, Q}=\overline{\mathbf{H}}_{Q} \mathbf{U}_{Q}^{(J)}
$$

where

$$
\mathbf{U}_{Q}^{(J)}=\left[\begin{array}{llll}
\mathbf{U}_{Q}(1) & \mathbf{U}_{Q}(2) & \cdots & \mathbf{U}_{Q}(J-1)
\end{array}\right]
$$

is a $(2 M+Q-1) \times Q(J-1)$ matrix. Suppose $J$ is sufficiently large so that $\mathbf{U}_{Q}^{(J)}$ has full rank $2 M+Q-1$. Then the rank of $\mathbf{Y}_{J, Q}$ is exactly $2 M+Q-1$, i.e., $\mathbf{Y}_{J, Q} \mathbf{Y}_{J, Q}^{\dagger}$ has exactly $L$ zero eigenvalues. This property, however, is no longer true when the block synchronization is not perfect. When a timing offset $d$ is present, the matrix in (7) becomes

$$
\mathbf{Y}_{J, Q}^{(d)}=\left[\begin{array}{llll}
\mathbf{Y}_{Q}^{(d)}(1) & \mathbf{Y}_{Q}(2) & \cdots & \mathbf{Y}_{Q}^{(d)}(J-1)
\end{array}\right]
$$

where

$$
\mathbf{Y}_{Q}^{(d)}(n)=\left[\begin{array}{llll}
\overline{\mathbf{y}}_{0, Q-1}^{(d)}(n) & \overline{\mathbf{y}}_{1, Q-2}^{(d)}(n) & \cdots & \overline{\mathbf{y}}_{Q-1,0}^{(d)}(n)
\end{array}\right]
$$

and

$\bar{y}_{k l}^{(d)}(n)=\left[\left[\left[\mathbf{y}_{M}^{(d)}(n-1)\right]_{M}^{-k+1}\right]^{T} \mathbf{y}_{c p}^{(d)}(n)^{T} \quad\left[\left[\mathbf{y}_{M}^{(d)}(n)\right]_{M+l}^{1}\right]^{T}\right]^{T}$.

The following theorem presents the theoretical foundation for the proposed algorithm.

Theorem 1: Assume each channel coefficient $h_{k}, 0 \leq k \leq$ $L$, is an independent complex Gaussian random variable and each element of $\mathbf{s}(n)$ is i.i.d. and selected from a finite constellation. Then with probability one there exists a sufficiently large $J$ such that the following statement on the matrix $\mathbf{Y}_{J, Q}^{(d)}$ defined in (9) is true with probability one.

$$
\begin{aligned}
& \text { The number of zero eigenvalues of } \mathbf{Y}_{J, Q}^{(d)} \mathbf{Y}_{J, Q}^{(d) \dagger} \\
& =(2 M+L+Q-1)-\operatorname{rank}\left(\mathbf{Y}_{J, Q}^{(d)} \mathbf{Y}_{J, Q}^{(d) \dagger}\right) \\
& = \begin{cases}L & \text { if } d=0 \\
\max \{L-|d|-2(Q-1), 0\} & \text { if } d \neq 0\end{cases} \\
& \text { Proof: See [10]. }
\end{aligned}
$$

We should note that a necessary condition (but not sufficient) for $\mathbf{U}_{Q}^{(J)}$ having full rank is [1]

$$
J \geq 2+\frac{2 M-1}{Q} .
$$

Although (10) is not sufficient, the probability that $\mathbf{U}_{Q}^{(J)}$ having full rank is usually very high in the simulation shown in Section IV. Inequality (10) also suggests, when the repetition is chosen sufficiently large (e.g., $Q=2 M-1$ ), the proposed algorithm can work with only three received blocks in absence of noise!

In presence of noise, the optimal $d$ can be taken to be the one which minimizes the sum of the smallest $L$ eigenvalues of $\mathbf{Y}_{J, Q}^{(d)} \mathbf{Y}_{J, Q}^{(d)}{ }^{\dagger}$. The proposed algorithm can be summarized as follows.

\section{Algorithm 1:}

1) Choose the repetition index $Q \geq 1$ and the number of collected blocks $J \geq 3$ so that (10) is satisfied.

2) Collect $(J+1) P$ consecutive received samples and form the matrix $\mathbf{Y}_{J, Q}^{(d)}$ as in Eq. (9) for each $d \in[-P / 2, P / 2)$.

3) Perform eigen-decomposition on the matrix $\mathbf{Y}_{J, Q}^{(d)} \mathbf{Y}_{J, Q}^{(d) \dagger}$ and take the $L$ smallest eigenvalues $\sigma_{L,(d)}^{2} \geq \sigma_{L-1,(d)}^{2} \geq$ $\cdots \geq \sigma_{2,(d)}^{2} \geq \sigma_{1,(d)}^{2} \geq 0$.

4) Calculate the cost function $\lambda(d):=\sum_{k=1}^{L} \sigma_{k,(d)}^{2}$, and decide the estimated timing offset $\hat{d}=$ $\arg \min _{-\frac{P}{2} \leq d<\frac{P}{2}} \lambda(d)$.

\section{A. Comparisons with a Previously Reported Algorithm}

In [2], a block synchronization algorithm was proposed by Negi and Cioffi based on the estimated rank of the autocorrelation matrix of received blocks. The number of received blocks therefore is required to be at least the block size (i.e., $J \geq M+L)$ in order to obtain an accurate statistics. As a comparison with (10), we find that the required number of received blocks of the proposed algorithm is smaller than the Negi-Cioffi algorithm when the repetition index $Q$ is chosen greater than unity.

Another important difference between the proposed algorithm and the Negi-Cioffi algorithm is that the latter requires the effective channel length $L_{0}$ to be strictly less than the cyclic prefix length $L$. When the channel order is equal to cyclic prefix length $\left(L=L_{0}\right)$, the Negi-Cioffi algorithm fails to work while our proposed algorithm still works properly.

\section{Simulation Results}

In this section, we conduct computer simulations to evaluate the performances of the proposed algorithms under different repetition indices $Q$ and compare them with that of the NegiCioffi algorithm [2]. In all simulations, the number of data samples per block is chosen as $M=8$ and the length of cyclic prefix is $L=4$ (which implies $P=12$ ). The precoder is chosen as $\mathbf{R}=\mathbf{I}_{M}$. The constellation of data samples is QPSK. Simulations are conducted with more than 3,000 independent 4-tap Rayleigh channels (i.e., $L_{0}=3$ ) with power-delay profile $[0.0-0.9-1.7-2.6](\mathrm{dB})$. The block synchronization error rate is calculated by averaging results for all channels. Note that a cyclic prefix of length $L=4$ allows a maximum number of channel taps to be five to avoid interblock interference. The reason why we chose only 4-tap channels is for proper comparison with the Negi-Cioffi algorithm [2]. This choice also implies that both $\hat{d}=0$ and $\hat{d}=-1$ will be considered a correct answer. In the plots, $E_{s}=E\left[\left|s_{k}(n)\right|^{2}\right]$ and $N_{0}=E\left[|e(n)|^{2}\right]$.

Figure 2 depicts the block synchronization error rate performance when 22 received blocks are available. We see that when $Q=1$, the proposed algorithm works properly in the high-SNR region, but with a rather unsatisfactory performance. The proposed algorithm with $Q=2$ has a much better performance and has a roughly 5 -dB gain over the Negi-Cioffi algorithm. When $Q$ is chosen as an even larger integer, the improvement of the block synchronization error rate performance is insignificant. In the high-SNR regions, the performances of the cases $Q=3,4$ are even worse than that of the case $Q=2$. 


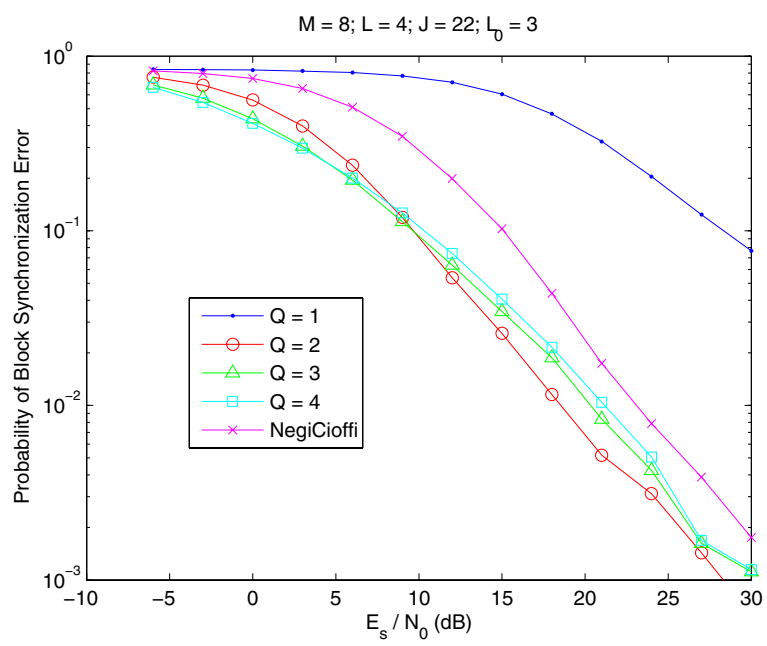

Fig. 2. Blind block synchronization error rate performance when 22 blocks are available

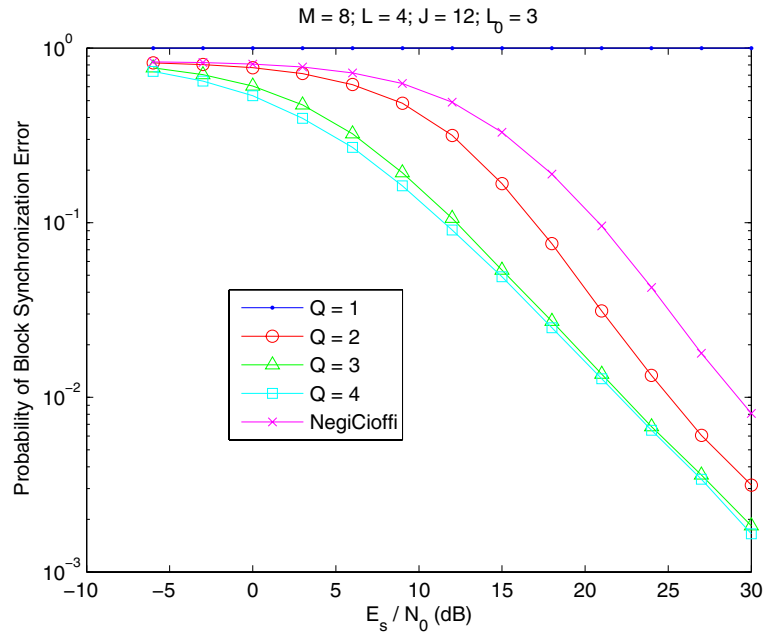

Fig. 3. Blind block synchronization error rate performance when 12 blocks are available

In Figure 3 we perform the simulation with a smaller number of received blocks $J=12$. In this case the proposed algorithm with $Q=1$ does not work since inequality (10) is no longer satisfied. The proposed algorithm with $Q=2$ still has a roughly 4-dB advantage over the Negi-Cioffi algorithm. When $Q$ is chosen as 3 , the block synchronization error rate performance has an obvious further improvement over the case where $Q=2$. But when $Q$ increases from 3 to 4 , the improvement in performance is limited.

In Figure 4 we use an even smaller number of received blocks $J=8$. In this situation the Negi-Cioffi algorithm is no longer working, neither is the proposed algorithm with $Q \leq 2$. However, the proposed algorithm with $Q=3,4$ works with a reasonably satisfactory performance. This demonstrates the superiority of the proposed algorithm with a limited amount of available received data, if the repetition index $Q$ is properly chosen. This feature of the proposed algorithm is especially favorable in an environment of fast-varying channels (e.g., wireless channels).

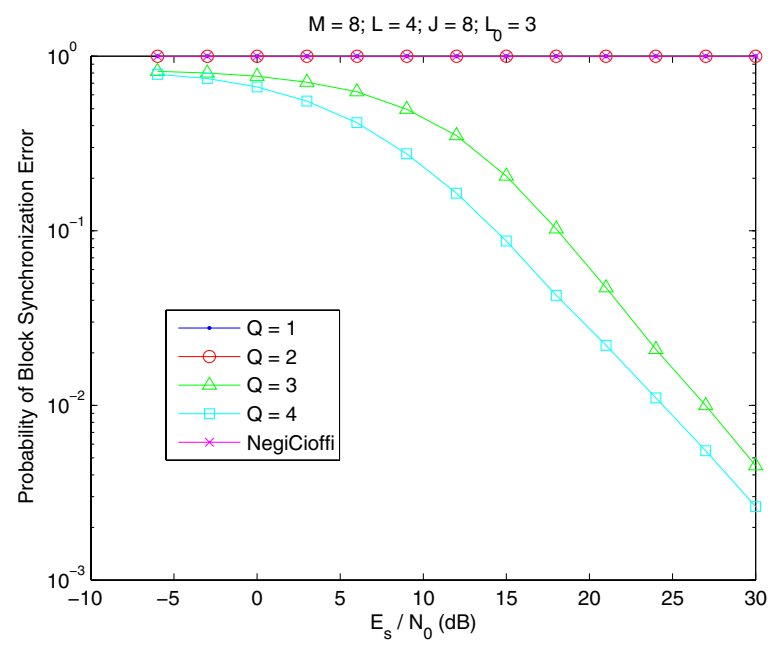

Fig. 4. Blind block synchronization error rate performance when 8 blocks are available

\section{COnCLUSions}

In this paper, a new blind block synchronization algorithm in cyclic prefix systems is proposed. It can be directly applied to blind symbol synchronization problem in OFDM systems. The proposed algorithm is capable of blindly recovering the correct block boundaries using much less received data than previously reported algorithms. This feature makes the proposed algorithm more promising in an environment of fastvarying channels. Simulation results not only demonstrate the capability of the proposed algorithm to work properly with limited amount of received data but also reveal significant improvement in block synchronization error rate performance over previously reported algorithms.

\section{REFERENCES}

[1] B. Su and P. P. Vaidyanathan, "Subspace-based blind channel identification for cyclic prefix systems using few received blocks," IEEE Trans. $S P$, vol. 55 , no. 10 , pp. 4979-4993, Oct. 2007.

[2] R. Negi and J. M. Cioffi, "Blind OFDM symbol synchronization in ISI channles," IEEE Trans. Commun., vol. 50, no. 9, pp. 1525-1534, Sept. 2002.

[3] B. Su and P. P. Vaidyanathan, "New Algorithms for Blind Block Synchronization in Zero-Padding Systems," Proc. of ICASSP 2007, Honolulu, Hawaii.

[4] B. Muquet, M. de Courville, and P. Duhamel, "Subspace-based blind and semi-blind channel estimation for OFDM systems," IEEE Trans. Signal Process., vol. 50, no. 7, pp. 1699-1712, Jul. 2002.

[5] D. Lee and K. Cheun, "A new symbol timing recovery algorithm for OFDM systems," IEEE Trans. Consumer Elecron., vol 43, pp. 767-775, Aug. 1997.

[6] A. Armada and M. Ramon, "Rapid prototyping of a test modem for terrestrial broadcasting of digital television, "IEEE Trans. Consumer Electron., vol. 43, pp. 1100-1109, Nov. 1997.

[7] J. van de Beek, M. Sandell, and P. O. Borjesson, "ML estimation of time and frequency offset in OFDM systems," IEEE Trans. Signal Processing, vol. 45, pp. 1800-1805, July 1997.

[8] A. Palin and J. Rinne, "Enhanced symbol synchronization method for OFDM system in SFM channel," in Proc. IEEE Globecom, Sydney, Australia, Nov. 1998, pp. 2788-2793.

[9] P. P. Vaidyanathan, Multirate systems and filter banks, Prentice-Hall, 1993.

[10] Proof of Theorem 1, http://www.systems.caltech.edu/borching/proof_cp.pdf (for reviewers only). 